\title{
RESULTS OF THE ENUMERATION OF COSTAS ARRAYS OF ORDER 29
}

\author{
Konstantinos DraKaKis \\ School of Electrical, Electronic \& Mechanical Engineering \\ University College Dublin, Belfield, Dublin 4, Ireland \\ FRANCESCO IORIO \\ Autodesk Research \\ 210 King Street East, Toronto, Ontario M5A 1J7, Canada \\ SCOTT RICKARD \\ School of Electrical, Electronic \& Mechanical Engineering \\ University College Dublin, Belfield, Dublin 4, Ireland \\ JOHN WALSH \\ Department of Computer Science \\ Trinity College Dublin, College Green, Dublin 2, Ireland
}

(Communicated by Andrew Klapper)

\begin{abstract}
The results of the enumeration of Costas arrays of order 29 are presented: except for 16 arrays out of a total of 164, all other arrays found are accounted for by the Golomb and Welch construction methods. These 16 arrays, however, cannot be considered to be new, as they were discovered in the past through a semi-empirical technique. The enumeration was performed on several computer clusters and required the equivalent of 366.55 years of single CPU time.
\end{abstract}

\section{INTRODUCTION}

Costas arrays [3] are square arrays of dots/1s and blanks/0s, such that there exists exactly one dot per row and column (that is, they are permutation arrays), and such that a) no four dots not lying on a straight line form a parallelogram, b) no four dots lying on a straight line form two equidistant pairs, and c) no three dots lying on a straight line are equidistant. They were first proposed by J.P. Costas in 1965 in the context of SONAR detection in a GE technical report [1], and later in 1984 in a journal publication [2].

There are two known algebraic construction techniques for the production of Costas arrays: the Golomb and Welch methods. Both are based on the theory of finite fields and were introduced and proven in 1984 by S. Golomb and H. Taylor $[6,8]$. These methods successfully construct $n \times n$ Costas arrays for infinitely many, but not all, orders $n$, and they remain the only generally applicable construction techniques for Costas arrays available today.

2000 Mathematics Subject Classification: Primary: 05A15, 05B10; Secondary: 05A05, 11B50. Key words and phrases: Costas arrays, enumeration, order 29, Golomb method, Welch method. Konstantinos Drakakis and Scott Rickard are also affiliated with the Claude Shannon Institute (www.shannoninstitute.ie), as well as with UCD CASL (casl.ucd.ie). 
In this work we present the results of the enumeration of Costas arrays of order 29. These results come approximately eight months and 2.5 years after the results of the enumeration of Costas arrays of order 27 [5] and 28 [4], respectively. This presentation is meant to be read as a supplement to [4]: the reader is referred therein, in particular, for all relevant definitions not included here.

\section{Definition AND BASICS}

Let us begin with the definition of a Costas function/permutation $[2,3]$ :

Definition 1. Let $[n]:=\{1, \ldots, n\}, n \in \mathbb{N}$ and consider a bijection $f:[n] \rightarrow[n]$; $f$ is a Costas permutation iff

$$
\begin{aligned}
& \forall i, j, k \text { such that } i, j, i+k, j+k \in[n]: \\
& \qquad f(i+k)-f(i)=f(j+k)-f(j) \Rightarrow i=j \text { or } k=0 .
\end{aligned}
$$

This condition is known as the Costas property.

Permutations correspond to permutation arrays by setting the elements of the permutation to denote the positions of the (unique) 1 in the corresponding column of the array, counting from top to bottom: $f(i)=j \Leftrightarrow a_{j, i}=1$. It is customary to represent the 1's of a permutation array as "dots" and the 0's as "blanks". The terms "array" and "permutation" will henceforth be used interchangeably.

The Costas property is invariant under rotations of the array by $90^{\circ}$, horizontal and vertical flips, and flips around the diagonals, hence a Costas array gives birth to an equivalence class (EC) that contains either eight Costas arrays, or four if the array happens to be symmetric; in the latter case, we say the EC is symmetric. When presenting the results of the enumeration, we will give the lexicographically minimal representative from each EC for brevity; the members of the EC of a Costas array are often referred to as its polymorphs.

There exist two algebraic methods for the construction of Costas arrays, known as the Golomb and Welch methods [3, 6, 8, 7], each actually comprising several submethods: relevant to this work are Golomb methods $G_{0}, G_{2}$, and $G_{3}$, and Welch methods $W_{0}$ and $W_{2}$. A further semi-empirical method relying on these two, known as the Rickard method and consisting of two submethods, is currently the only additional method that has successfully led to the discovery of four previously unknown Costas arrays, namely two of order 29 (through the Rickard Welch $R W_{0}$ submethod), one of order 36, and one of order 42 [9]. A summary of all these techniques can be found in [4]. Any additional Costas array not resulting from these techniques will be characterized as sporadic.

As the Rickard method is not very well known, it is perhaps worth offering a brief description here. Appending a blank row to an exponential $W_{1}$ array and then cyclically shifting its rows any number of times results in a non-square array with the Costas property. If a column is then appended on either side with a single dot corresponding to location of the blank row, a new permutation array is obtained, which may have the Costas property (the same effect can be achieved by appending a blank column to a logarithmic $W_{1}$ array and then cyclically shifting its columns). Similarly, appending both a blank row and a blank column to a $G_{2}$ array and then cyclically shifting both its rows and its columns independently any number of times results in a new square array with the Costas property, which can furthermore be turned into a permutation array through the placement of a new dot at the intersection of the blank row and column, and which may have the Costas property. 


\section{ENUMERATION RESULTS FOR ORDER 29}

The enumeration found in total 164 Costas arrays, divided into 23 ECs, 5 of which are symmetric. More specifically, breaking down this set per construction method (descriptions of which can be found in [4]), there are:

- $4 G_{0}$-arrays in 1 symmetric EC, constructed in $\mathbb{F}(29)$;

- $64=8^{2}=\phi^{2}(30) G_{2}$-arrays in 10 ECs (4 symmetric), constructed in $\mathbb{F}(31)$;

- $24 G_{3}$-arrays in $3 \mathrm{ECs}$, constructed in $\mathbb{F}(32)$;

- $24 W_{0}$-arrays in $3 \mathrm{ECs}$, constructed in $\mathbb{F}(29)$;

- $32 W_{2}$-arrays in $4 \mathrm{ECs}$, constructed in $\mathbb{F}(31)$;

- $16 R W_{0}$-arrays in $2 \mathrm{ECs}$, constructed in $\mathbb{F}(29)$.

The lexicographically minimal polymorphs are shown in Table 2, while the two $R W_{0}$-arrays are plotted in Figure 1 . As was the case for order $n=28$, none of the arrays found is sporadic. The total runtime of the project on a single CPU was recorded to be approximately 366.55 years (about 5.2 times as long as the enumeration of Costas arrays of order 28 recorded in [4]), but, due to high parallelization of the tasks, the real time required was approximately 230 days, between 2010/06/08 and $2011 / 01 / 24$. This shows that, on average, approximately 568 processors were used throughout the run (though, briefly, at peak times, simultaneous use of up to about 2,000 processors was recorded). The memory and storage requirements of the code used were minimal.

Forbidden positions for Costas arrays of order 29 are shown in Figure 2: they correspond to positions in which none of the arrays carries a dot. The resulting cross-shaped region is similar to the cross-shaped regions appearing in the forbidden positions of orders 25 and 27 [5]. There were no forbidden positions in order 28: the cyclic shift property of $W_{1}$-arrays, present in all orders $n=p-1, p$ prime (hence 28 as well), implies that the dots of a single such array along with all of its cyclic shifts will span all elements of the array, leaving no gaps.

\section{A BRIEF DESCRIPTION OF THE THE PROCEDURE FOLLOWED}

Our code was essentially the same as the one used by our group for the enumeration of Costas arrays of order 28 [4]. The project was run on several clusters in Ireland:

- UCD's Phaeton cluster, managed by UCD IT Services, and featuring Intel Xeon E5450 and E5540 Quad Core CPUs, at 3.00GHz and 2.53GHz, respectively. We were allocated a maximum of $200 \mathrm{CPUs}$, while jobs were submitted through a custom-made task-farming Python PBS script.

- UCD's SenseTile cluster, managed by members of UCD CASL, and featuring AMD Opteron 8360SE Quad Core CPUs at 2.51GHz We were allocated 78 reserved CPUs, and jobs were submitted through a custom-made task-farming Python SGE script.

- The Stokes supercomputer of the Irish Centre for High End Computing (ICHEC), featuring Intel Westmere Xeon E5650 Hex Core CPUs at 2.66GHz. We used a recorded total of 1.6 million CPU hours allocated through a Class A ICHEC grant. Bundles of jobs were submitted to be run using Stokes's builtin task-farming facilities, each bundle allocated 288 CPUs and a walltime of half a week (84 hours). 

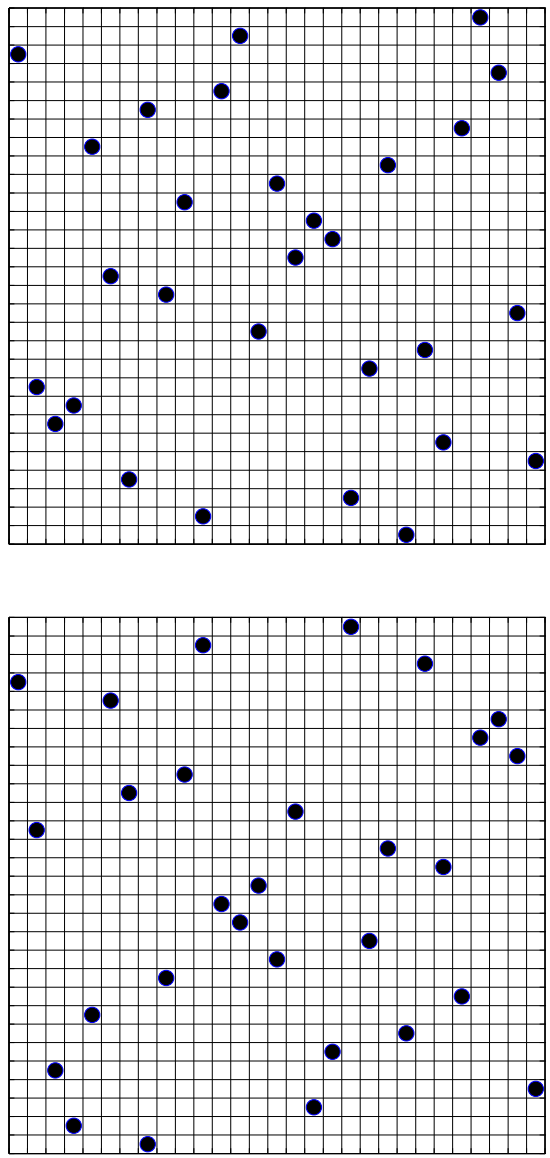

Figure 1. The two lexicographically minimal Welch Rickard Costas arrays of order 29, corresponding to permutations 32123 228152661611285218101412132720929192471417 25 (top) and 412252822510292092161715191127241 1813233142176826 (bottom)

- Grid-Ireland, administered by members of Trinity College Dublin's (TCD) School of Computer Science and Statistics, and featuring a variety of CPUs located all across Ireland. Jobs were initially submitted through the DIANE/GANGA queue submission system, but subsequently a new grid job framework and workflow was developed to comprehensively handle bounded job submission, job status monitoring with error handling, and output retrieval. A walltime of three days was individually applied to each job.

- Lonsdale, administered by the Trinity Centre for High Performance Computing (TCHPC), and featuring AMD Opteron 2350 Quad Core CPUs running at $2.00 \mathrm{GHz}$. Jobs were submitted through custom scripts handling SLURM, the built-in job submission system. 


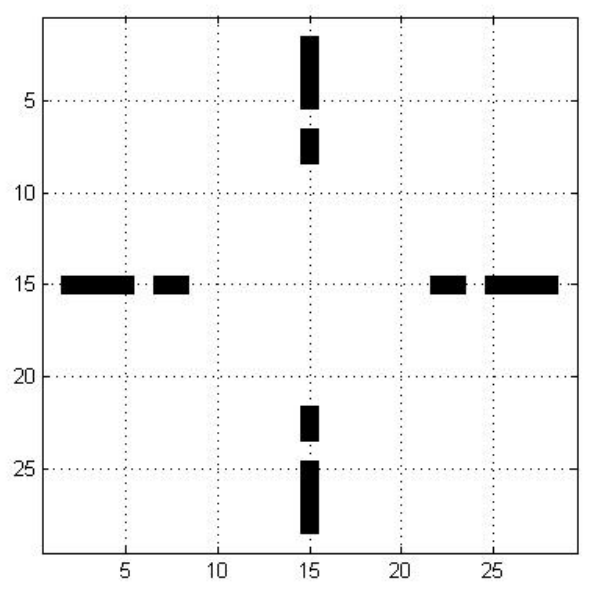

FiguRe 2. Forbidden positions for Costas arrays of order 29

\begin{tabular}{|l|r|r|r|r|}
\hline Cluster & Jobs run & $\%$ & CPU years run & $\%$ \\
\hline \hline Stokes (ICHEC) & 106,724 & 54.22 & 217.37 & 59.30 \\
\hline Phaeton (UCD) & 63,473 & 32.25 & 79.72 & 21.75 \\
\hline SenseTile (CASL) & 13,744 & 6.98 & 27.12 & 7.40 \\
\hline Grid-Ireland & 10,358 & 5.26 & 36.88 & 10.06 \\
\hline TCHPC (TCD) & 2,540 & 1.29 & 5.46 & 1.49 \\
\hline \hline Total & 196,839 & 100 & 366.55 & 100 \\
\hline
\end{tabular}

TABle 1. Statistics (number of jobs run and CPU time used) per cluster

Specific statistics per cluster are shown in Table 1. Runtimes of individual jobs, when the latter are lexicographically sorted, are shown in 3 (top), while the corresponding histogram is shown in Figure 3 (bottom). In the top figure, 13 individual ramp-shaped parts are clearly discernible, corresponding to the starting integer (2 to 14) of the permutations tested. The high spikes in the second ramp (corresponding to starting integer 3) are due to the slowest processors of Grid-Ireland.

\section{Conclusion}

Costas arrays of order 29 have been enumerated: there are 164 Costas arrays divided into 23 equivalence classes, five of which are symmetric. Had it not been for the publication of [9] in 2004, two equivalence classes would have been new/sporadic, but, as it stands, no new/sporadic Costas arrays were found, as was the case for order 28.

\section{ACKNOWLEDGMENTS}

The successful completion of this project would not have been possible without the assistance of numerous individuals. The authors would like to acknowledge, in particular: Fred Clarke and Ruth Lynch (UCD IT Services) for helping with the runs on Phaeton; Chris Bleakley (UCD, School of Computer Science and Informatics) 

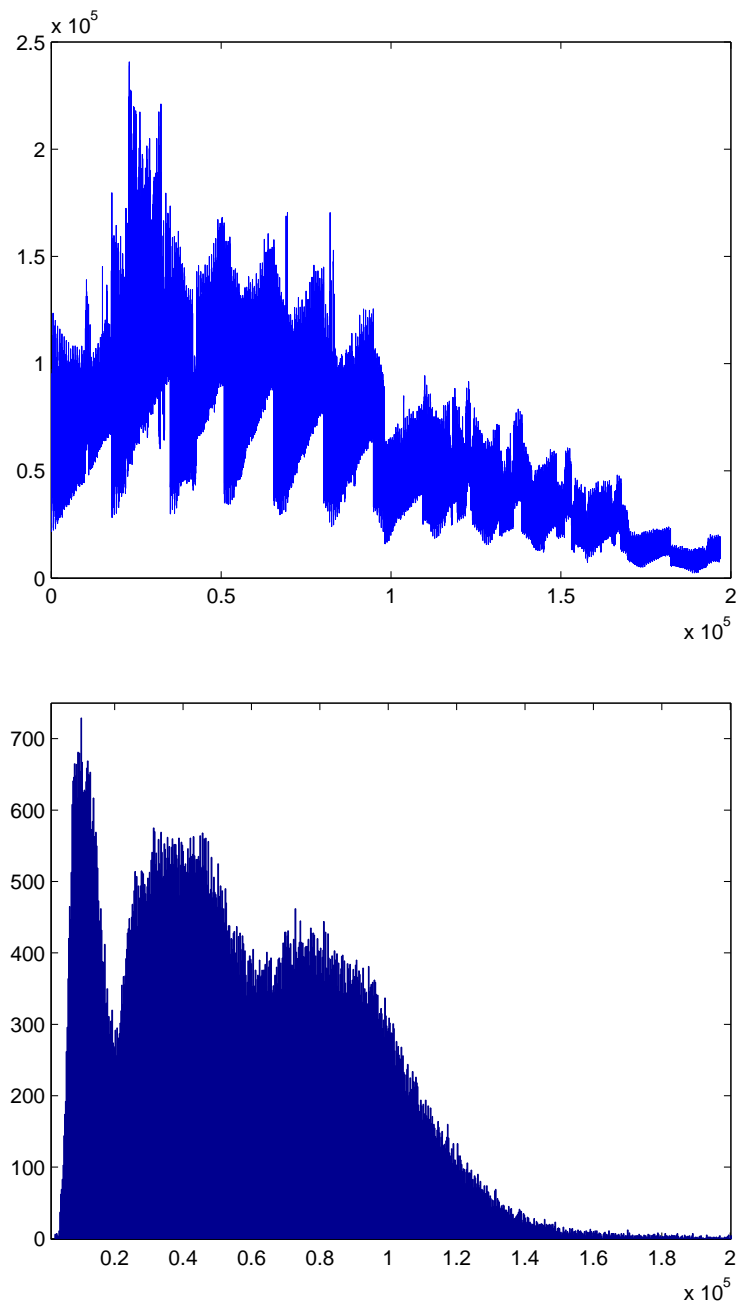

FiguRE 3. Top: runtimes of jobs arranged in lexicographical order. Bottom: the histogram of runtimes of the various instances of the project (1000 bins); outlier runtimes of more than 200,000s are not shown.

for granting us the use of the SenseTile cluster; ICHEC for approving Class A grant ndmat007a, and Dr. Michael Browne, in particular, for his support and for many helpful discussions.

This material is based upon works supported by the Science Foundation Ireland under Grant No. 05/YI2/I677, 06/MI/006 (Claude Shannon Institute), and 08/RFP/MTH1164.

\section{REFERENCES}

[1] J. P. Costas, Medium constraints on SONAR design and performance, in "Technical Report Class 1 Rep. R65EMH33," GE Co., 1965; A synopsis of this report appeared in the EASCON Convention Record, (1975), 68A-68L. 


\begin{tabular}{|c|c|}
\hline 714 & $G_{3}$ \\
\hline 2221237281417129 & $G_{3}$ \\
\hline 11325234721202216299661551217102614182882824192713 & $G_{3}$ \\
\hline 11229325142619282718247461121151622108221752023139 & $G_{2}$ \\
\hline 1172213192324271182515287186261221352016149210429 & $G_{0} / \mathrm{s}$ \\
\hline 122014162522231326127282142911171569981851924310272 & $W_{0}$ \\
\hline 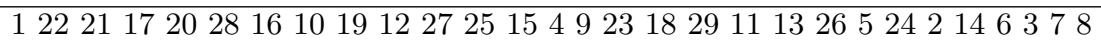 & $W_{0}$ \\
\hline $125222313261272821429111715698 \quad 81851924310272201416$ & $W_{0}$ \\
\hline 7212719102624523201416 & $G_{2} / \mathrm{s}$ \\
\hline 2826182515161928241272392927213114141310151722620 & $W_{2}$ \\
\hline 611272825813166 & $G_{2}$ \\
\hline 22810125182220239272971312263381914112161552416174 & $G_{2}$ \\
\hline 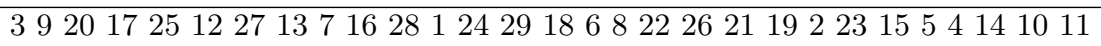 & $G_{2}$ \\
\hline 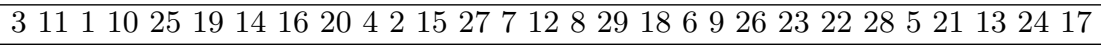 & $G_{2} / \mathrm{s}$ \\
\hline 321232281526616112852218101412132720929192471141725 & $R W_{0}$ \\
\hline 4122528225102920921617151911272411813233142176826 & $R W_{0}$ \\
\hline 426201756614101039132911242221162328727121525182198 & $G_{2}$ \\
\hline 6191220252218826171292892413141621123371052742115 & $W_{2}$ \\
\hline 62623285827161614718421122021539102419292517111322 & $G_{2} / \mathrm{s}$ \\
\hline 7132175272821862015192212418291426431023251116129 & $W_{2}$ \\
\hline 7202882515114232722141612613181726222911922451910321 & $G_{2} / \mathrm{s}$ \\
\hline 82210192512282017213111371814272126299924155642316 & $G_{2}$ \\
\hline 10272885312182242315201329192121242617117256149 & $W_{2}$ \\
\hline
\end{tabular}

TABLE 2. The lexicographically minimal polymorphs of Costas permutations of order 29 , shown along with the method producing them. A "/s" indicates the EC is symmetric.

[2] J. P. Costas, A study of detection waveforms having nearly ideal range-doppler ambiguity properties, Proc. IEEE, 72 (1984), 996-1009.

[3] K. Drakakis, A review of Costas arrays, J. Appl. Math., 2006, 32 pp.

[4] K. Drakakis, F. Iorio and S. Rickard, The enumeration of Costas arrays of order 28 and its consequences, Adv. Math. Commun., 5 (2011), 69-86.

[5] K. Drakakis, S. Rickard, J. Beard, R. Caballero, F. Iorio, G. O'Brien and J. Walsh, Results of the enumeration of Costas arrays of order 27, IEEE Trans. Inform. Theory, 54 (2008), $4684-4687$.

[6] S. W. Golomb, Algebraic constructions for Costas arrays, J. Combin. Theory Ser. A, 37 (1984), 13-21.

[7] S. W. Golomb, The $T_{4}$ and $G_{4}$ constructions for Costas arrays, IEEE Trans. Inform. Theory, 38 (1992), 1404-1406.

[8] S. W. Golomb and H. Taylor, Constructions and properties of Costas arrays, Proc. IEEE, 72 (1984), 1143-1163.

[9] S. Rickard, Searching for Costas arrays using periodicity properties, in "IMA International Conference on Mathematics in Signal Processing at The Royal Agricultural College," Cirencester, UK, 2004.

Received for publication January 2011.

E-mail address: Konstantinos.Drakakis@ucd.ie

E-mail address: Francesco.Iorio@autodesk.com

E-mail address: Scott.Rickard@ucd.ie

E-mail address: John.Walsh@cs.tcd.ie 